\title{
Uncovering Spatial Structures of Regional City Networks from Expressway Traffic Flow Data: A Case Study from Jiangsu Province, China
}

\author{
Wenqian Ke ${ }^{1,2}$ (D), Wei Chen ${ }^{3,4, *}$ (D) and Zhaoyuan Yu ${ }^{5,6}$ \\ 1 Institute of Geography, Fujian Normal University, Fuzhou 350007, China; wqke2005@163.com \\ 2 Key Laboratory of Humid Subtropical Eco-geographical Process, Ministry of Education, \\ Fujian Normal University, Fuzhou 350007, China \\ 3 Key Laboratory of Regional Sustainable Development Modeling, Institute of Geographic Sciences and \\ Natural Resources Research, Chinese Academy of Sciences, Beijing 100101, China \\ 4 School of Resources and Environment, University of Chinese Academy of Sciences, Beijing 100049, China \\ 5 College of Geography Science, Nanjing Normal University, Nanjing 210023, China; \\ yuzhaoyuan@njnu.edu.cn \\ 6 Jiangsu Center for Collaborative Innovation in Geographical Information Resource Development \\ and Application, Nanjing 210023, China \\ * Correspondence: geochw@163.com
}

Received: 30 July 2017; Accepted: 26 August 2017; Published: 29 August 2017

\begin{abstract}
On the basis of the "space of flows" theory, city networks emphasizing factor connectivity and spatial connection have become a core perspective for regional spatial relationships. They provide a context for discussing the spatial structures of city networks and a scientific basis for making regional development policies. Relying on expressway traffic flow data from Jiangsu Province in 2014, this study describes macro-spatial patterns and hierarchical structures of city networks, and uses the Walktrap algorithm of community detection to execute space divisions and elucidate potential spatial connection structures in the city networks. In comparison with other algorithms, the Walktrap algorithm demonstrates significant adaptation and stability to the short expressway traffic flows with the characteristics of strong network density and sparse node distribution. The results indicate that the macro-spatial patterns of Jiangsu's city networks have clear regional differences. The cities with relatively dense spatial linkages are distributed along the Yangtze River banks, and many different sub-network systems have also developed internally. Cities and linkage axles have clear hierarchical structures. City hierarchies have certain spatial couplings with the cities' intrinsic social economic attributes and geographical locations. The linkage axles hierarchy has clear spatial interactions and superposition with geographical distance. The community detection algorithm identified six spatially connected "city communities", the Suzhou-Wuxi-Changzhou Community (SWCC), the Nanjing-Zhenjiang-Yangzhou-Taizhou Community (NZYTC), the Nantong-Yancheng Community (NYC), the Lianyungang Community (LYGC), the Huai'an-Suqian Community (HSC), and the Xuzhou Community (XZC). The community spatial metaphors had four aspects. First, trans-prefectural linkages were formed through spatially integrated effects of metropolis regions. Second, some communities share the same boundaries with their prefecture level administrative units; this reveals that significant administrative regional economies still exist in contemporary Jiangsu. Third, several cities located in the marginal areas of prefectures and captured by the powerful center cities in neighboring prefectures have been absorbed into the communities of neighboring prefectures. Fourth, the two cities Jiangyin and Jingjiang, divided into different administrative districts, have switched status opposite city community divisions.
\end{abstract}

Keywords: city network; regional division; traffic flow; community detection; Jiangsu Province; China 


\section{Introduction}

Since the late 20th century, as globalization and informatization has accelerated and grown, the network society began to rise. In this context, the concept of "space of flows" has gradually replaced "space of place" as the basic theory for reviewing regional space interactions. Research on regional spatial structures has also transformed from the central place model of rating scale to the city network model of factor relations [1-3]. Given the daily innovations in information technology, communication technology and means of transport, constant access to various intelligent terminals to the Internet, and improvements in infrastructure, various virtual and real world factor flows have gradually become normal and frequent. This has formed closer inter-city connections. The city hierarchy and function divisions have profoundly changed. These changes are manifested in the interactive influences of "space of place" and "space of flows". The spatial structures of inter-city connections do not simply and strictly follow the "core-hinterland" neighborhood characteristic anymore; instead, cities can maintain close connections with a non-neighboring city beyond the scope of traditional hinterlands [4]. Inter-city relationships have also gradually transformed from static, closed, and hierarchical-centered models to dynamic, open, and flat multi-centered network models. Similarly, regional spatial structure theories have been redefined and modified within this context described. For example, factor connectivity weakens geographic proximity, and the relationship theory supersedes the location theory [5]. Concurrently, from the perspective of relational geography, the concept of "city network" indicating city connections with various factor flows may well explain inter-city linked characteristics. These characteristics generally agree with the previously mentioned trend, and a series of concepts have been successively proposed, such as world city network [6-8], global city region [9] and mega city region $[10,11]$. Researching the spatial structures, organization models, and evolution process of inter-city connection using factor flows of different types and scales, from the network perspectives, could effectively elucidate the operating mechanism of a city. Furthermore, this type of research can clarify a city status within the present urban systems, which has become a frontier subject in urban geography.

Obtaining factor flow data of different types and scales enriches empirical research on the spatial structures of city networks. This empirical research encompasses transport and information infrastructure, enterprise organizations, and social cultural relationships. Transport infrastructure research commonly includes analyzing air, railway, and highway traffic flow. Data are primarily the statistics for connections and flows between large and medium cities, as counted by traffic and transportation departments [12-14]. Additional breadth to the field will be gained from researching the spatial characteristics of city networks across different spatial scales using information and communication flows in information facilities, enterprise organization relation flows in the productive service industry [7,15-17], and knowledge and culture flows in social and cultural relationships [18,19].

Research on the spatial characteristics of city networks has included three aspects. First, most research methods have adopted simplex or multiple types of solid road networks to calculate traffic accessibility, introduced gravity models to interactively calculate city attribute indexes, and simulated interactions of social economic systems [20,21]. Second, some research has combined abstract nodes and node-connecting edges into a "network graph" using graph theory, and adopted complicated network indexes to measure topology and quantify the nodal relationships [22,23]. Third, some scholars have weighted values to represent the strength of connections between nodes or sides to create clustering indices; these clusters can then be used to analyze network structures, most commonly with community detection or cohesive subgroup algorithms [24,25].

However, restricted by the scarcity of relational data and difficulty in obtaining such data, most factor flow data remain insufficient in terms of sample quantity, continuity, timeliness, and traceability. This problem is an "Achilles' Heel" for researching city networks, and restricts the effective elucidation of city network spatial structures and their governing laws [26]. Identifying the effective space division of city networks and types of spatially linked structures can be accomplished by searching continuous, massive, and dynamic factor flow data. This type of analysis will provide (1) a new understanding of 
existing city network relationships, and (2) a mechanism for introducing a network analysis model that gives concurrent consideration to the uniform expression of network topology and city connections.

Expressways are a convenient connection for passenger and cargo flows over short distances. Their factor flow provides concurrent consideration to plan and immediacy characteristics, has distinctive space dependence and distance attenuation characteristics, and well describes functional interactions inside complete areal systems. Therefore, they can represent important characteristics for surveying the connection structures of city hierarchies and geographical distances in the spatial interactions of city networks. Therefore, this study surveys the macro-spatial patterns and hierarchical structures of city networks, with the support of the daily average expressway traffic flow data in 2014 for Jiangsu Province. A community detection algorithm is used to conduct space division and type recognition of city networks, and reveals the potential spatial structures of inter-city connections. The purpose of this paper is as an empirical study of diversification and quantitative analysis relating to city networks and their spatial structures. In short, the objectives of this study are to provide (1) a basis for innovative models or divisions of spatial structures of city networks under the perspective of "space of flows", and (2) a scientific basis for forming regional development strategies or spatial planning policies.

\section{Research Area, Data, and Methods}

As illustrated in Figure 1, we introduce a methodological scheme that summarizes the steps of the procedure used, which consists of key steps: (1) introducing the basic characteristics of Jiangsu Province, China, processing the basic expressway traffic flow data, thereby constructing the OD flow matrix; (2) utilizing the Jenks natural breaks method and the K-means clustering method to analyze the hierarchical structure of cities and city-dyads, respectively (as mentioned above, we also mapped the macro-spatial patterns of city networks); (3) using the Walktrap algorithm to detect city communities for city networks; (4) describing the spatial connectivity of each community in the aforementioned city networks; and (5) identifying spatial organization models of city communities in city networks.

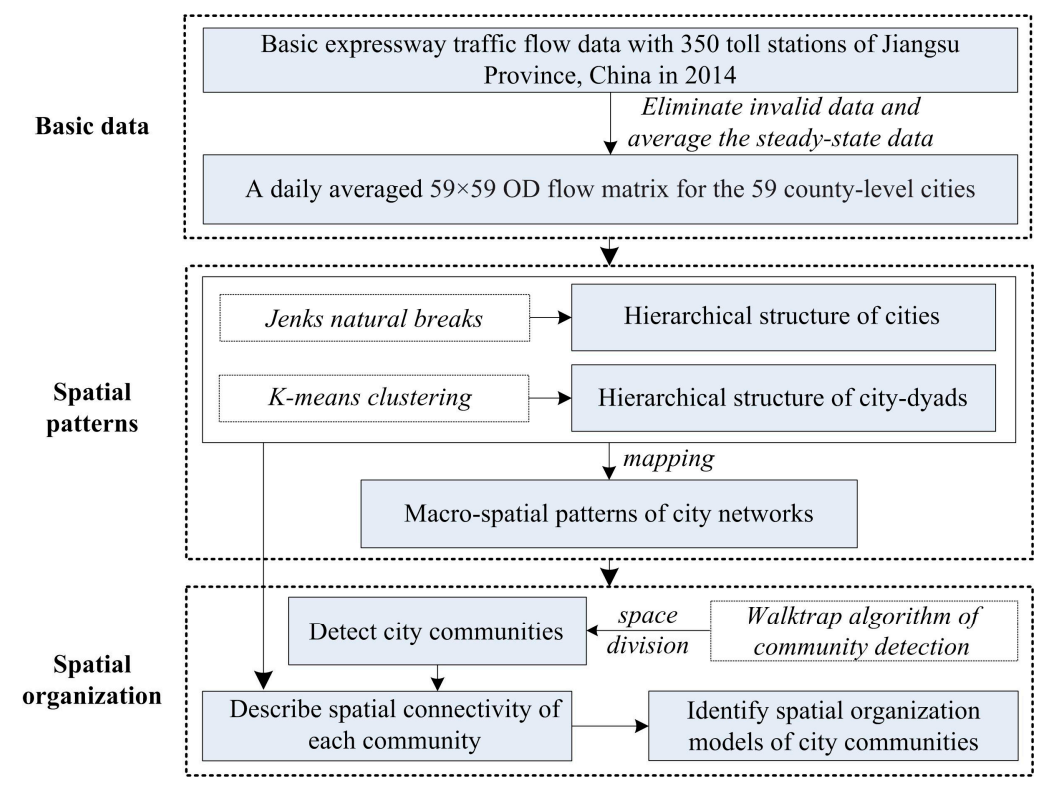

Figure 1. Workflow diagram of the methodology used in this study.

\subsection{Research Area}

Jiangsu Province is located on the coast of eastern China, and governs 13 prefecture-level cities with 63 county-level units, including county, county-level city, and municipal districts. Within the 
province, nine prefecture-level cities, Nanjing, Wuxi, Changzhou, Suzhou, Nantong, Yancheng, Yangzhou, Zhenjiang, and Taizhou, belong to the Yangtze River Delta Economic Zone. Among the nine prefecture-level cities in Jiangsu Province, there are seven cities with a population of more than one million, with Nanjing as the largest with 7.36 million residents. In general, larger cities are mainly concentrated in southern Jiangsu, including as Suzhou, Wuxi, Changzhou, Zhenjiang, and Nantong. Simultaneously, more and medium sized cities are located in northern Jiangsu, except for the cities of Xuzhou, Huai'an and Yancheng. This regional disparity has long been a significant phenomenon in Jiangsu Province. The landforms of Jiangsu Province are primarily plains, and it is relatively short in the east-west direction, narrow and long in the south-north direction. Approximately $370 \mathrm{~km}$ of the Yangtze River crosses the province from west to east, dividing it into south and north areas. The presence of the Yangtze River blocks a convenient transportation channel for communication between south and north Jiangsu. The economic status of the province has been harmed by the disconnection between the south and north, and economic wealth progressively decreases from south to north. From the economic conditions, the province can be generally divided into three blocks: Southern Jiangsu, including Suzhou, Wuxi, Changzhou, Zhenjiang, and Nanjing; Mid-Jiangsu, including Nantong, Taizhou, and Yangzhou; and Northern Jiangsu, including Yancheng, Lianyungang, Huai'an, Suqian, and Xuzhou. To break the Yangtze River's separation of south and north Jiangsu and facilitate economic development, the province has accelerated and improved the construction of the expressway network and channels crossing the Yangtze River. By the end of 2014, Jiangsu ranked first among provincial units nationwide in expressway network density. It had formed the "four vertical, four horizontal and four connection" pattern, constructed up to 10 channels crossing the Yangtze River, with 350 toll stations with origin destination (OD) flow between toll station nodes, essentially covering the entire region (Figure 2).

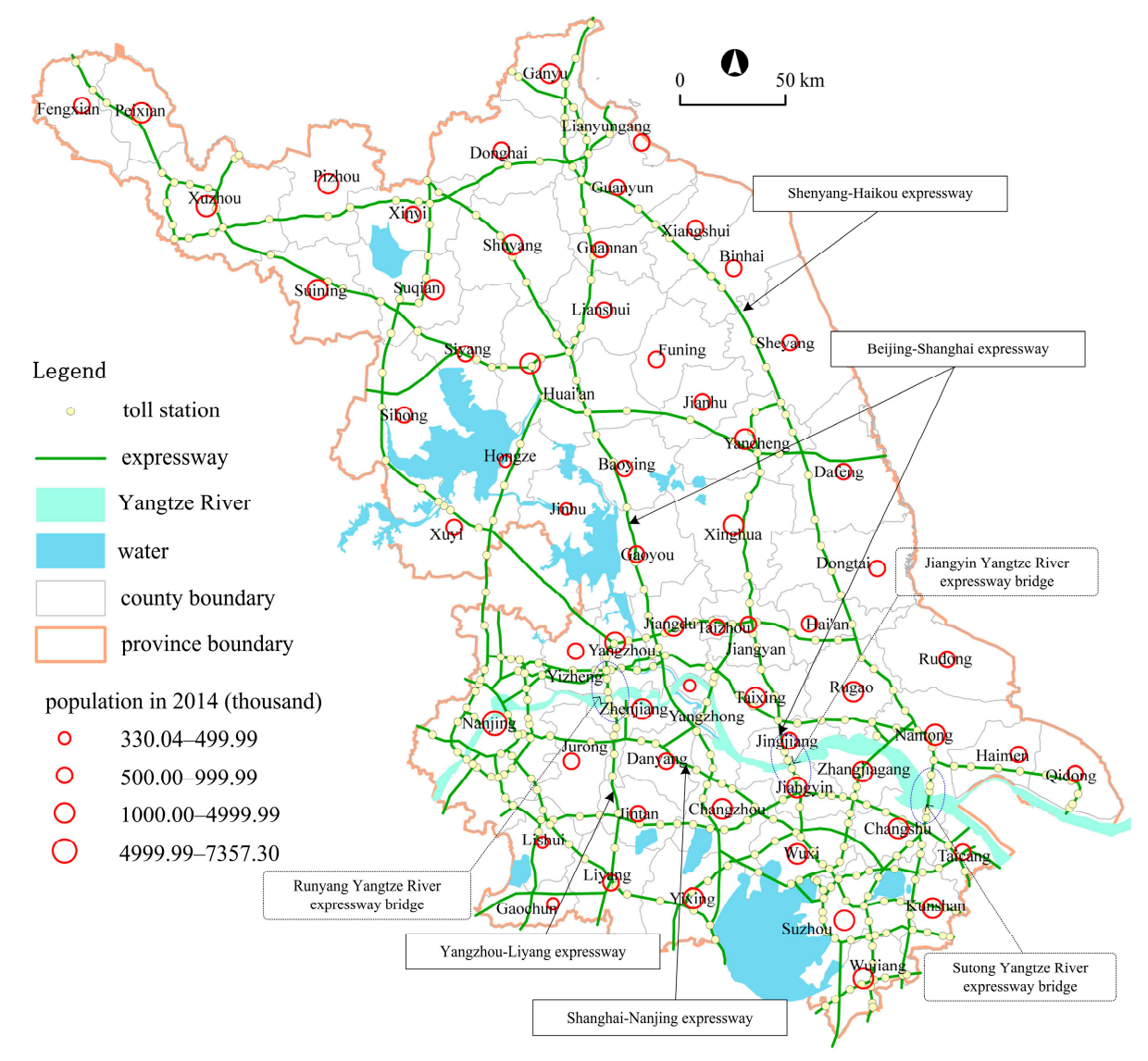

Figure 2. Spatial distribution of expressway toll stations in Jiangsu province during the research period. 


\subsection{Data Source and Processing}

The traffic flow data adopted in this paper were sourced from daily traffic flow on expressways of Jiangsu Province in 2014. Every recorded entry indicates the Provincial ETC number of each vehicle, serial number of toll gates for entrance and exit, and time of entrance and exit stations. During analysis, this study summarized each recorded entry based on the same "entrance toll station number-exit toll station number" at daily time and county-level city spatial scales; potentially invalid data were eliminated prior to use. From these steps, we obtained an OD flow matrix of continuous and daily traffic for 59 county-level cities in Jiangsu province for 365 days from 1 January 2014 to 31 December 2014 (within the research period, the flow data from the expressway toll station nodes for Gaochun County, Rudong County, Funing County, and Jinhu County were unavailable, so the flows for these counties were not considered, and the city networks comprised of the other 59 county-level cities were analyzed here). In the original data, days prior and subsequent to certain holidays and festivals, including New Year's Day, Spring Festival, Tomb-sweeping Day, Labor Day, Dragon Boat Festival, Mid-Autumn Festival, and National Day, showed short-term fluctuations in expressway traffic flow. Furthermore, the expressways were toll-free during the Spring Festival, Tomb-sweeping Day, Labor Day, and National Day, so small passenger cars with less than seven seats were released directly and no data exists for these vehicles in this period of time. In comparison, the daily flow on days other than holidays and festivals generally maintained a relatively steady level. Therefore, to elucidate the daily spatial structures of city networks within one year, this study eliminated the traffic flow data on holidays and festivals, and the surrounding dates. Data were eliminated from 1-5 January 2014; 16 January-25 February 2014; 3-9 April 2014; 29 April-5 May 2014; 29 May-4 June 2014; 4-10 September 2014; 24 September-14 October 2014; 30-31 December 2014, which was 117 days (For three-day holidays and festivals, the data for seven days, including the holidays and festivals, and the days before and after them, were eliminated; for National Day, data for 21 days, including days prior to and after, were eliminated; for the Spring Festival, data for 40 days, including days prior to, after, and the full Spring Festival were eliminated; because New Year's Day crossed calendar years the prior day data were missing, and traffic flow on 30-31 December 2014 clearly rose, data prior to these days in the following year were eliminated as part of the New Year's Day holiday period). Next, the steady-state OD flow traffic for the remaining 248 days was averaged, and the data used for analysis were incorporated in a daily averaged $59 \times 59$ OD flow matrix for the 59 county-level cities.

\subsection{Community Detection}

In complex networks, a community, sometimes referred to as a cluster or a module, is a fundamental qualitative concept that is defined by a group of densely connected nodes sparsely connected to nodes of other groups in the network. As a unique feature of real-world networks, community structure has been a straightforward indicator to graphically illustrate both global and local heterogeneity of vertices and edge distributions. The community structure detection technique identifies similar groups of nodes in a network. More importantly, the community detection process has proven effective in describing a variety of different phenomena in real-world contexts, ranging from natural sciences to social sciences to engineering. Concurrently, many methodologies have been developed for community detection, and mainstream algorithms include Girvan-Newman [27], Fast-greedy [28], Walktrap [29], Multi-level [30], Label Propagation [31], and Infomap [32].

Among these algorithms, Pons and Latapy (2006) proposed the well-known Walktrap algorithm, which utilizes hierarchical clustering with distance based on random walks. The fundamental assumption of the Walktrap algorithm is that random walks tend to get trapped into densely connected subgroups of the network, which correspond to communities. Therefore, the algorithm measures the structural similarity between vertices and communities by defining a distance that is calculated from the probabilities that the random walk moves from one vertex to another in a fixed number of steps. Nodes that are within the same community should have short distances between them, and, 
conversely, nodes from different communities would have large distances. The distance $r_{i j}$ between node $i$ and $j$ is defined as:

$$
r_{i j}=\sqrt{\sum_{k=1}^{n} \frac{\left(P_{i k}^{t}-P_{j k}^{t}\right)^{2}}{d(k)}}
$$

where $P_{i k}^{t}$ is the probability to go from node $i$ to node $k$ in $t$ steps of a random walk; $P_{j k}^{t}$ is the probability to go from node $j$ to node $k$ in $t$ steps of a random walk. The transition probability $P_{i j}$ at each step is defined as $P_{i j}=\frac{A_{i j}}{d(i)}$ where $A_{i j}$ is the value of adjacency matrix $A$ and $d(k)$ is the degree of node $k, d(k)=\sum_{j} A_{k j}$. Similarly, the distance between two communities can also be consistent with the definition of previous one.

The algorithm proceeds as follows:

(1) The initial step separates each node into its own community.

(2) The two closest communities are then merged into a new community based on the distance between them.

(3) This procedure is iterated until a single community consisting of all nodes is formed.

(4) The dendrogram is finally formed and the community partition is finished.

Additional details regarding the implementation of Walktrap can be found in the original work [29].

In spatial networks, the community detection method can be an ideal tool to reveal inherent structures. Compared to other algorithms, Walktrap has displayed greater robustness and accuracy in weighted networks and can be computationally more efficient $[33,34]$. On one hand, Jiangsu Province's intercity road linkages demonstrate the characteristics of strong network density and sparse node distribution; in this regard, the Walktrap algorithm would perform better identifying potential community structures. On the other hand, however, as intercity road linkages represent remarkable spatial dependence and distance decay, the Walktrap algorithm via short random walks therefore becomes an ideal technique to explore spatial structures and organization in city networks. Here, we see a city as a node of networks and the linkage strength of a city as the weight of a node. Thus, the network edges can be expressed via the intercity linkages, and edge weights can be expressed via the linkage frequencies between cities. All data processing and algorithm implementations were performed on the R platform.

\section{Macro-Spatial Patterns and Hierarchical Structures of City Networks}

\subsection{Macro-Spatial Patterns of City Networks}

The spatial patterns of city networks are formed through mapping overall architectural structure relationships of nodes and axles in regional space. The patterns of Jiangsu's city networks are plotted by combining the hierarchy of cities and city-dyads' connection axles, as shown in Figure 3. According to this figure, cities with relatively good space connection and network density were distributed along the Yangtze River banks, including Southern Jiangsu and Mid-Jiangsu, and extended northward to the north boundary of Yangzhou, Taizhou, and Nantong. Multiple different sub-network systems developed internally. Suzhou, Wuxi, and Changzhou in Southern Jiangsu had the highest network density in the whole region, and formed essentially uniform spatial structures, that is, the linkage frequencies were equal. Unlike Suzhou, Wuxi, and Changzhou, the connections between the core of Nanjing and network with Zhenjiang, Yangzhou, and Taizhou were not as close. In Mid-Jiangsu, the networks with Nantong as the core were characterized by a prefecture-level city scope, with relatively close connections with Suzhou and Changshu southward and Yancheng northward. In Northern Jiangsu, the regions with ordinary network connections and network intensity including Yancheng, Huai'an, Lianyungang and Xuzhou, and their networks basically covered the scope extending to the 
boundary of their prefecture-level cities. Suqian did not develop clear network patterns, and had extremely sparse connections with its county-level city.

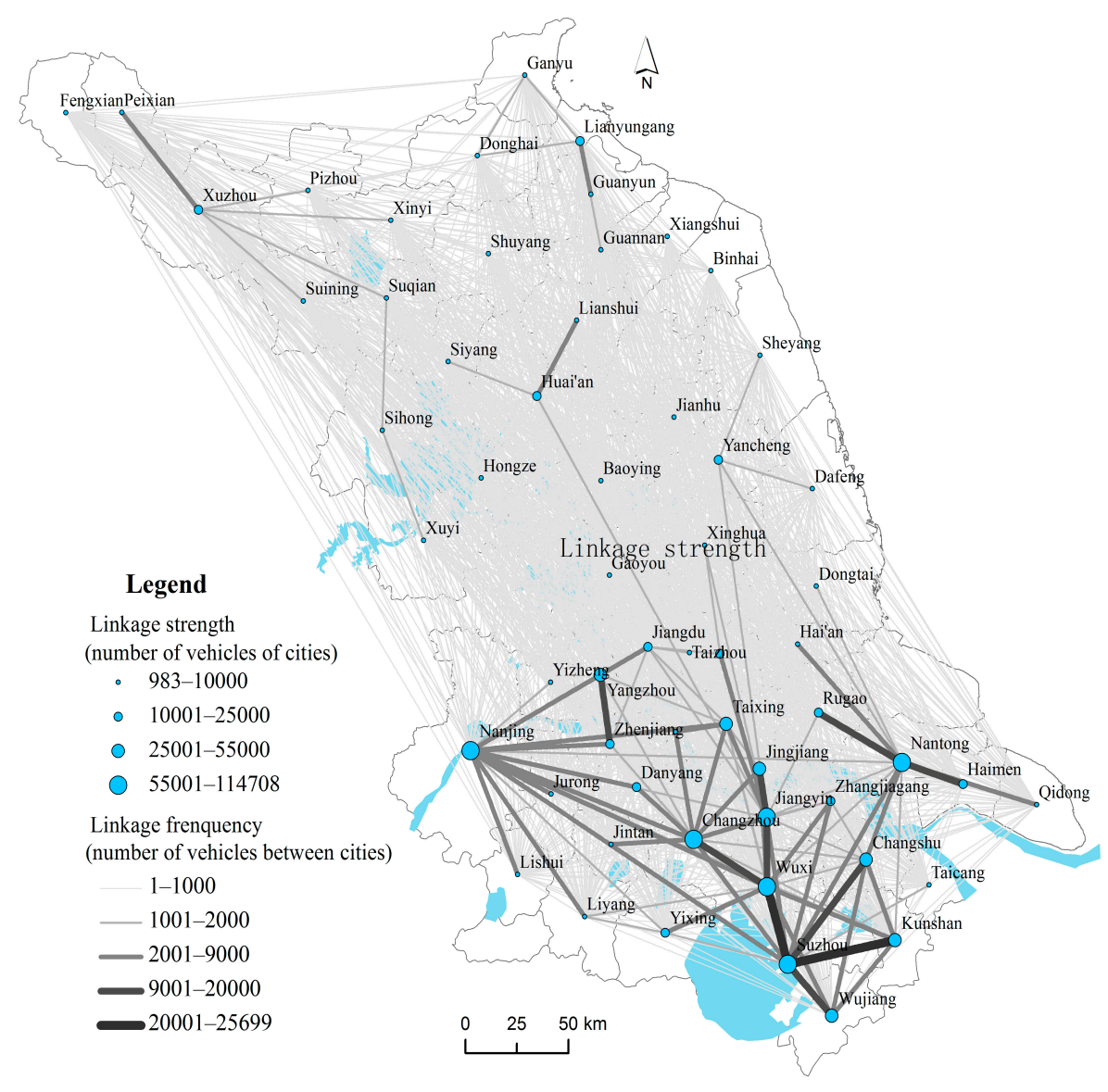

Figure 3. Macro-spatial patterns of city networks in Jiangsu Province.

Generally, the spatial patterns of Jiangsu's city networks represented by expressway traffic flow show clear differences between the south and north. The overall patterns show that the best-developed cities are located at the boundary of this province; well-developed cities are located along main trunk expressways, while the worst-developed cities are located inside the province and regions north of the Yangtze River. The cities located at the boundary of the province include Suzhou (Wuxi), Nanjing, Nantong, and Xuzhou; the cities located at trunk expressways are mainly distributed along the Shanghai-Nanjing Expressway, Beijing-Shanghai Expressway, Shenyang-Haikou Expressway, and Shanghai-Xi'an Expressway. In comparison, cities located in the province hinterlands are mainly distributed in the vast region of Northern Jiangsu. Such distribution characteristics show that Jiangsu Province does not act as a complete economic regional unit; instead, it belongs to both the Yangtze River Delta Economic Zone, with Shanghai at the core, and the Huaihai Economic Zone, with Xuzhou at the core. From the perspective of the metropolis circle, Jiangsu could be divided into three different metropolis circles. The first is the Suzhou-Wuxi-Changzhou Metropolis Circle with Shanghai as the core, including Suzhou, Wuxi, Changzhou, Nantong, and Yancheng, and the southeast direction as the major gravitational direction. The second is Nanjing Metropolis Circle with Nanjing as the core, including Nanjing, Zhenjiang, Yangzhou, and Taizhou, and the southwest direction as the major gravitational direction. The third is Huaihai Metropolis Circle with Xuzhou as the core, including Xuzhou, Huai'an, Suqian, and Lianyungang, and the northwest direction as the major gravitational direction. As previously discussed, the gravitational direction of different economic zones and metropolis circles is outward, and the dominant direction has relatively great differences. Therefore, 
the existing urban distribution pattern of Jiangsu Province can be characterized as a typical external expansion and, due to relatively weak internal spatial organization, this province shows relatively unrestricted qualities.

\subsection{Hierarchical Structure of Cities}

The gradation of different cities is a basic factor that distinguishes spatial structure types of city network. From the analysis on the hierarchy of cities with the Rank-Size Rule, proposed by Zipf in 1949 [35], the urban system of cities follows the Pareto distribution. The Rank-Size Rule is expressed as $\ln P r=a-q \ln r\left(q=0.86, R^{2}=0.93\right)$, here $r$ indicates city rank, which is reflected by the linkage strength of city nodes, and the greater linkage strength of a city node, the higher of its rank; $P r$ indicates the linkage strength considering the total flow of city nodes; $a$ is a constant; and $q$ reflects the change in the result of node flow and scale (when $q=1$, it will presents a rank-scale distribution, when $q>1$, the scale level of nodes will differ greatly, and presents a primate city distribution, when $q<1$, the scale rating of nodes will present a logarithmic normal distribution). The importance of cities in the network differs significantly; the development of several large cities is relatively important, while that of small and medium cities is lower. On this basis, to survey the hierarchical difference in city status across the entire network, linkage strength is represented by number of vehicles of each city, and the city connection intensities were divided using natural breaks; the results are as shown in Table 1. "Core Cities" were those with spatial linkage strengths greater than 55,000 vehicles; these included Suzhou, Wuxi, Changzhou, Nanjing, Jiangyin, and Nantong. "Key Cities" were those with spatial linkage strengths of 25,000 55,000 vehicles; these included Kunshan, Changshu, Jingjiang, Yangzhou, Taixing, and Wujiang. Between the two types of cities, the riverside or close-to-Yangtze-River cities in Southern Jiangsu and Mid-Jiangsu and cities along the Shanghai-Nanjing Expressway and Shanghai-Jiangxi Expressway were dominant. The 12 "Important Cities" with spatial linkage strengths between 10,000 and 25,000 vehicles, included cities along the Yangtze River and the main urban areas and county-level cities in coastal and hinterland areas. The remaining cities were widely distributed and termed "general cities", with a spatial linkage strength of less than 10,000 vehicles. Cities with high hierarchy were relatively economically well-developed and wealthy, and were distributed along the Yangtze River, along the provincial boundary, or along the trunk expressways; the converse was true for cities of relatively low hierarchy. These results indicate the importance of city hierarchy on creating spatial coupling with social economic attributes and geographical location.

Table 1. Hierarchical distribution of cities.

\begin{tabular}{|c|c|c|}
\hline Hierarchy & Linkage Strength & Cities and Number in Hierarchical Class ( ) \\
\hline Core cities & $>55,000$ & Suzhou, Wuxi, Nanjing, Jiangyin, Nantong, and Changzhou (6 cities) \\
\hline Key cities & $25,000-55,000$ & $\begin{array}{c}\text { Kunshan, Changshu, Jingjiang, Yangzhou, Taixing, } \\
\text { and Wujiang (6 cities) }\end{array}$ \\
\hline Important cities & $10,000-25,000$ & $\begin{array}{l}\text { Zhenjiang, Rugao, Jiangdu, Huai'an, Zhangjiagang, Xuzhou, Yancheng, } \\
\text { Yixing, Haimen, Lianyungang, Jiangyan, and Danyang (12 cities) }\end{array}$ \\
\hline General cities & $<10,000$ & $\begin{array}{c}\text { Liyang, Hai'an, Guanyun, Yangzhong, Suqian, Jurong, Jintan, } \\
\text { and others (35 cities) }\end{array}$ \\
\hline
\end{tabular}

\subsection{Hierarchical Structure of Spatial Linkage Frequency}

Generally, the amount of traffic OD flow between cities can reflect the degree of closeness in spatial connections between them. Therefore, we set the number of vehicles between cities as the inter-city spatial connections to measure the "spatial linkage frequency" in this study. Based on the k-means clustering method [36], and as per city-dyads, the inter-city spatial linkage frequency was divided into five hierarchies, high strength, sub-high strength, medium strength, general strength, and low strength (Table 2). The city-dyads with high-strength connections were Suzhou-Wuxi and 
Suzhou-Kunshan; they have a spatial linkage frequency of above 20,000 vehicles, and have the closest connection within the entire regional scope. There were eight city-dyads with a sub-high-strength connection of 9000 20,000 vehicles; these included the city-dyads of Suzhou, Wuxi, and Changzhou, the urban areas of Nantong and respective county-level cities, and the city-dyads of Yangzhou-Zhenjiang crossing the Runyang Bridge and Jiangyin-Jingjiang crossing the Jiangyin Bridge. The medium spatial linkage frequency and general spatial linkage frequency were respectively 2000 9000 vehicles and 1000 2000 vehicles, corresponding to 39 city-dyads and 53 city-dyads, respectively. The city-dyads with low spatial linkage frequency, defined as 1 1000 vehicles, have reached a total of 1571, all of which were widely distributed across the entire region. Generally, the city-dyads connection axes showed a clear hierarchy difference in spatial linkage frequency. In addition, axle hierarchy and geographical distance had clear spatial interaction and superposition; city-dyads within a short distance of each other had extremely close connections, and presented a clear "localization" characteristic. This characteristic may provide a reference for describing the functional connection inside a relatively complete regional system.

Table 2. Hierarchical distribution of linkage frequency in city networks.

\begin{tabular}{|c|c|c|}
\hline Hierarchy & Linkage Frequency & City-Dyads and Number in Hierarchical Class ( ) \\
\hline High & $>20,000$ & Suzhou-Wuxi and Suzhou-Kunshan (2 city-dyad) \\
\hline Sub-high & $9000-20,000$ & $\begin{array}{l}\text { Suzhou-Changshu, Suzhou-Wujiang, Jiangyin-Jingjiang, } \\
\text { Nantong-Rugao, Yangzhou-Zhenjiang, Nantong-Haimen, } \\
\text { Wuxi-Changzhou, Wuxi-Jiangyin, and others (8 city-dyad) }\end{array}$ \\
\hline Medium & $2000-9000$ & $\begin{array}{l}\text { Wuxi-Jingjiang, Nanjing-Changzhou, Nantong-Changshu, } \\
\text { Wuxi-Yixing, Suzhou-Changzhou, Jiangyin-Taixing, } \\
\text { Yangzhou-Jiangdu, and others (39 city-dyad) }\end{array}$ \\
\hline General & $1000-2000$ & $\begin{array}{c}\text { Nanjing-Yixing, Jiangdu-Taixing, Wuxi-Changshu, } \\
\text { Changzhou-Zhangjiagang, Suqian-Sihong, Xuzhou-Suining, } \\
\text { Xuzhou-Pizhou, Lianyungang-Guannan, Yangzhou-Changzhou, } \\
\text { and others (53 city-dyad) }\end{array}$ \\
\hline Low & $1-1000$ & $\begin{array}{l}\text { Nantong-Taixing, Haimen-Qidong, Huai'an-Suqian, } \\
\text { Huaian-Hongze, Zhangjiagang-Jingjiang, Wuxi-Danyang, } \\
\text { and others (1571 city-dyad) }\end{array}$ \\
\hline
\end{tabular}

\section{Spatial Organizations of City Networks}

\subsection{Identification of City Communities Based on Network Connection}

To analyze the spatial structures of city networks, the Walktrap algorithm of community detection was adopted to execute the space division, as shown in Figure 4. Using this algorithm, a total of six "city communities" were obtained; they were respectively termed the Suzhou-Wuxi-Changzhou Community (SWCC), the Nanjing-Zhenjiang-Yangzhou-Taizhou Community (NZYTC), the Nantong-Yancheng Community (NYC), the Lianyungang Community (LYGC), the Huai'an-Suqian Community (HSC), and the Xuzhou Community (XZC). A "City Community" is divided based on the agglomeration and diffusion ability that represents the flow of production factors of inter-city functional connections. The community divisions result in four spatial metaphors. First is the integration effect, which is most clearly embodied by the integrated networks formed from the close combination via high-strength spatial connection of Suzhou, Wuxi, and Changzhou. Second is the administrative region effect, which is primarily embodied in the regions with ordinary internal connections and relatively low external connections, such as XZC and LYGC. These regions are located at boundaries, and central cities do not have sufficient agglomeration and diffusion ability, so can only maintain existing spatial connections within the scope of the prefecture-level city administrative region. Third is the capture effect, which is most clearly embodied in NZYTC, NYC, and HSC; one to two center cities with relatively strong 
agglomeration and diffusion ability appear between or among two or more neighboring regions. The center cities of other regions are relatively poorly developed and absorbed by the center cities with strong agglomeration ability, and thus become the influencers of their hinterlands. Fourth is the substitution effect. In these regions, the effect is an exchange between two cities, namely Jingjiang in SWCC and Jiangyin in NZYTC. In terms of administrative division, Jiangyin and Jingjiang belong to Wuxi and Taizhou respectively, but the converse is true in the division of city communities. This occurs primarily because of the extremely close connection of Jiangyin and Jingiang with SWCC. Among the top 10 cities connected with the two cities, more than $80 \%$ of cities connected with Jingiiang also belong to SWCC, while Jiangyin is connected with cities of different communities, and the connection is relatively disperse.

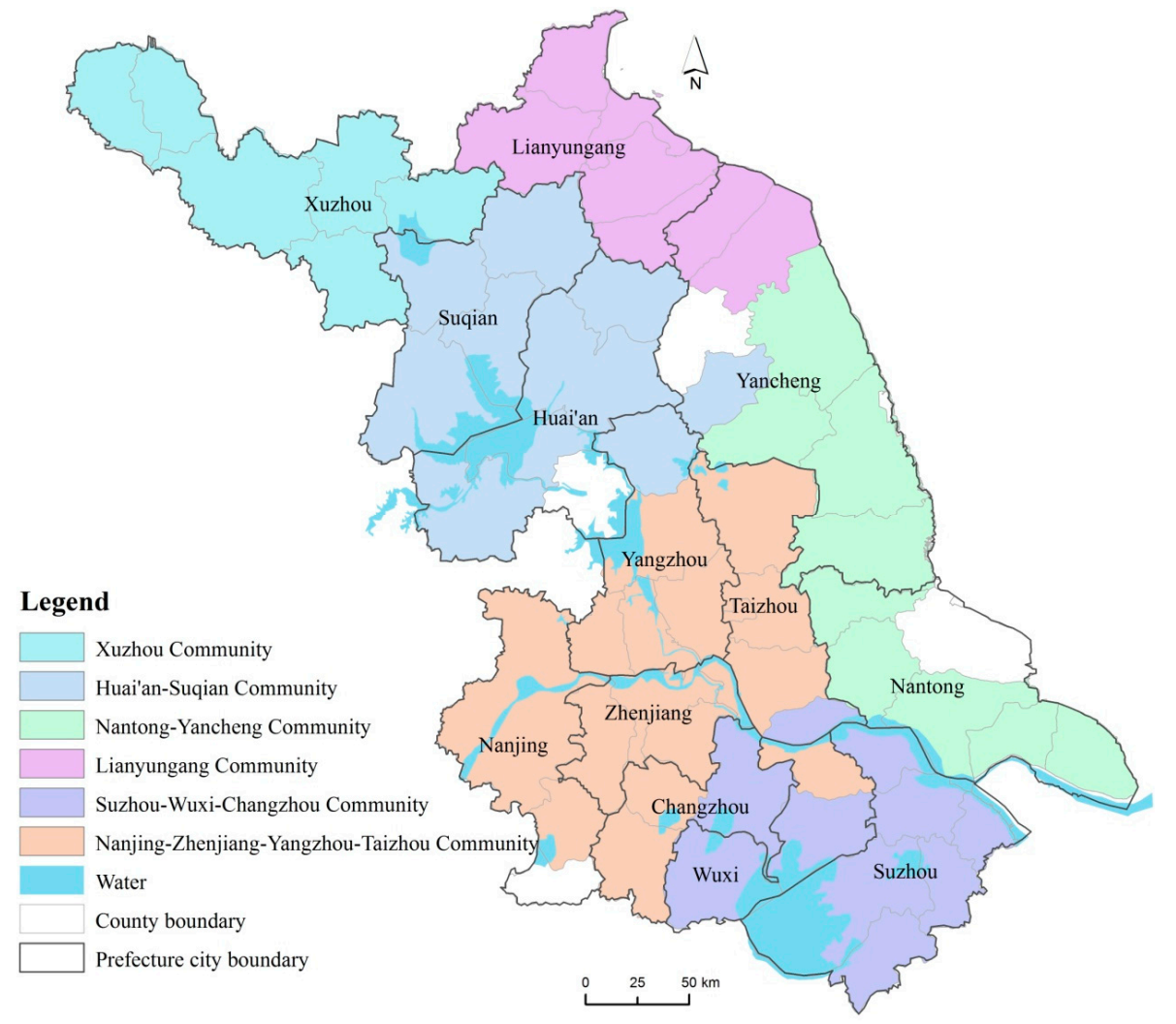

Figure 4. Community detection in the Jiangsu city networks.

\subsection{Spatial Connection Patterns of City Communities}

The internal spatial connection of city networks communities in Jiangsu Province is characterized by high internal connections between a few communities and relative sparse internal connection in other communities and a stronger intra-community internal connection than inter-community connection (Table 3). More than 75\% of spatial connections occur inside SWCC and NZYTC, the internal connections of these two communities accounts for $47.81 \%$ and $27.36 \%$ of all community internal connections. The NYC internal connection is also more than $10 \%(12.35 \%)$, but the connection of other communities is no more than $5 \%$. As shown in the proportion of community internal connections to the whole province, $62.96 \%$ of connections occur inside each community, and SWCC and NZYTC account for $30.10 \%$ and $17.23 \%$, respectively; therefore, they remain characterized by internal connections with other communities and SWCC and NZYTC are the communities with the highest network intensity and closest connection within the city networks of Jiangsu Province. This may be due to the two communities belonging to regions close to the Yangtze River or in Southern Jiangsu, which have 
well-developed economies, strong industrial growth, clear hierarchies and convenient access to the road network, and strong inter-city connection demands.

Table 3. Inner strength and proportion of each community in Jiangsu city networks.

\begin{tabular}{cccc}
\hline Community & Inner Strength & $\begin{array}{c}\text { Proportion of All } \\
\text { Community Strength (\%) }\end{array}$ & $\begin{array}{c}\text { Proportion of All Community } \\
\text { Inner Strength (\%) }\end{array}$ \\
\hline $\begin{array}{c}\text { Jiangsu province } \\
\text { All communities inner strength }\end{array}$ & 509,142 & 100 & - \\
XZC & 320,534 & 62.96 & 100 \\
HSC & 9712 & 1.91 & 3.03 \\
LYGC & 14,520 & 2.85 & 4.53 \\
NYC & 15,783 & 3.10 & 4.92 \\
NZYTC & 39,571 & 7.77 & 12.35 \\
SWCC & 87,707 & 17.23 & 27.36 \\
\hline
\end{tabular}

In combination with the city hierarchy divisions and city-dyads connection axle hierarchy, this study describes the spatial structure characteristics of each community's internal connections, as shown in Figure 5. Clearly, different communities have certain similarities and differences in terms of form and spatial organization. SWCC has the highest agglomeration ability in terms of internal organization network among the six communities. It has essentially formed block-shaped multi-level networks with Suzhou, Wuxi, and Changzhou as the core, and other cities as auxiliaries. NZYTC includes several cities of relatively high administrative level, such as Nanjing, Yangzhou, Zhenjiang, and Taizhou. However, the internal connections are not close enough. Here, only Nanjing and Jiangyin act as cores; they have high flow levels but different functions. Specifically, Nanjing tends to connect many cities, while Jiangyin is located at a border position, and plays a role as an external connection to portal cities. NYC, HSC, XZC, and LYGC are different from SWCC and NZYTC, because they have networks with single core cities. Clearly, in NYC, Nantong has stronger connection and contact with other cities inside this community, while the other three communities are relatively weak. LYGC is weaker than HSC and XZC based on the hierarchy of axle spatial linkage frequency. The neighboring county-level cities, Ganyu, Guannan, and Guanyun, are not weaker than the relatively high-level center city Lianyungang in terms of connections, although they are all generally weak.

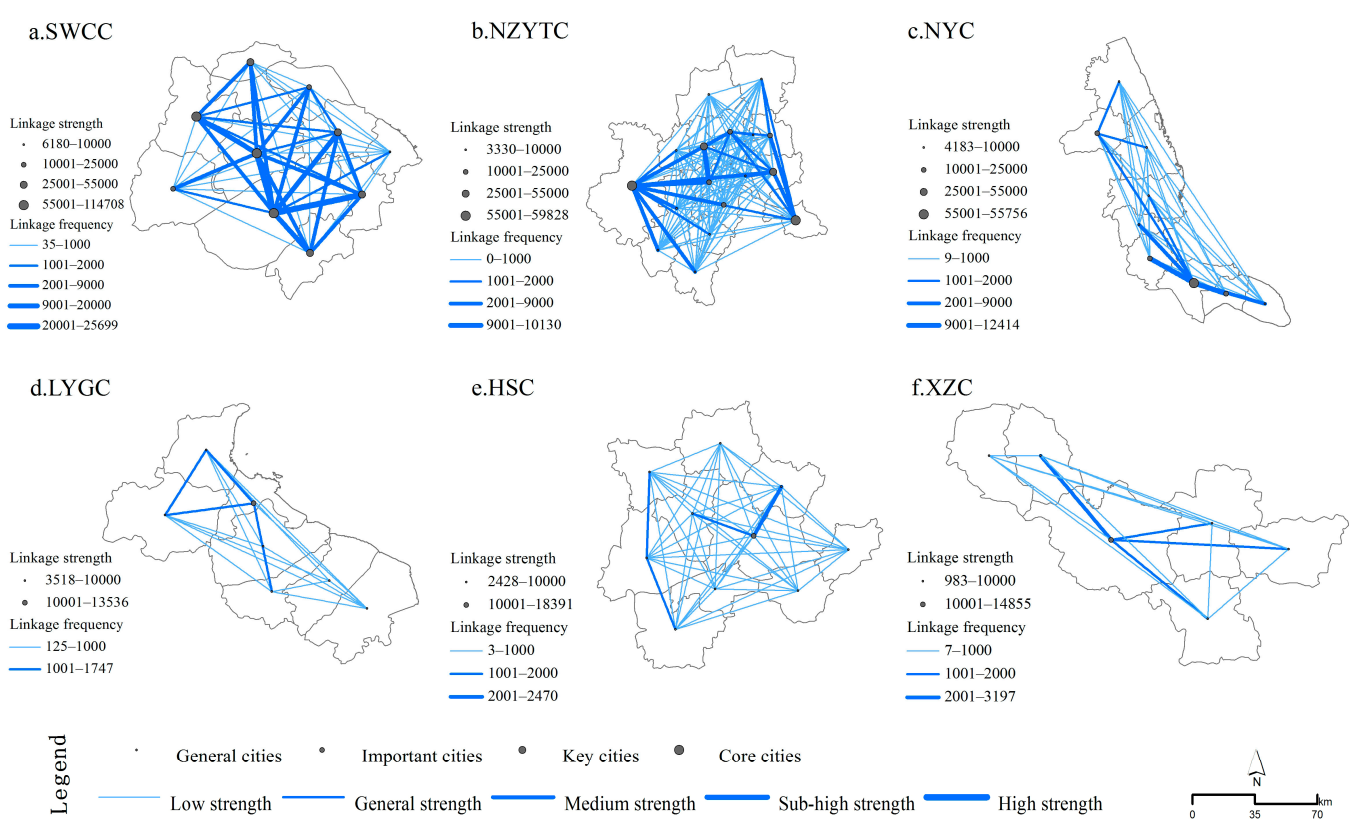

Figure 5. Spatial connectivity of each community in Jiangsu city networks. 


\subsection{Spatial Organization Models of City Communities}

Ideally, competition between cities will result in a hexagonal spatial form [5,37]. Based on this concept, the cities inside a community are extracted into two types. The first is a center city dominant formation and development of a surrounding community; the other type is a non-center city, which is an important part of the community. According to the similarity and difference characteristics in the internal connections between different communities, and the many combinations of center cities and non-center cities, four types of communities are shown in Figure 6. They are the polycentric development, dual-nuclei development, monocentric development, and equilibration development models.

$\begin{gathered}\text { Polycentric } \\ \text { development model }\end{gathered}$
$\begin{gathered}\text { Dual-nuclei } \\ \text { development model }\end{gathered}$

Figure 6. Four models forthe spatial connection of communities in Jiangsu city networks.

The first type polycentric development model is represented by SWCC, indicating the commonality of multiple center cities, and suggests that closely connected networks inside the community form easily. The dual-nuclei development model is represented by NZYTC, which has a center city located at the central position of social economic attributes, such as geometry, economy, population, and traffic, and one at a portal position, which jointly drive community development. Nanjing and Jiangyin are the center cities located at the center and portal positions respectively. The monocentric development model is represented by NYC, HSC, and XZC, which has a single center city located at relatively central position that drives the development of a whole community. Such communities are relatively unstable, and will likely further evolve to dual-core or multi-core communities. Especially for NYC and HSC, it is very probable that the two core cities of Nantong-Yancheng and Suqian-Huai'an to jointly dominate the development of community networks. The last model, equilibration development, occurs in the absence of a dominant core city; LYGC is a good representation of this model. Lianyungang is a relatively high-level city in this community, but in internal and external connection, Lianyungang does not occupy obvious advantages in comparison with other neighboring cities, so its function as a center city weak. This model generally occurs at the preliminary stage of network development, and there are multiple possibilities for such type to evolve to other types.

\section{Conclusions and Outlook}

\subsection{Conclusions}

The development of expressways has greatly influenced the accessibility and connectivity of cities and city-dyads within city networks, thereby influencing their location advantage and their positions in the urban relation network. This will change the spatial structures of entire city networks. A great deal of research has analyzed the topological properties or spatial structures of city networks by using transport infrastructure [12,13], yet literature regarding expressway traffic flow within regional city networks is limited. Therefore, it is important to describe the spatial structures of city networks based on the degree of connection closeness for expressway factor flows. Using factor relationships, 
and supported by daily average expressway traffic flow data for a full year in Jiangsu Province, this study describes the macro-spatial patterns and hierarchical structures of city networks. The Walktrap algorithm of community detection is used to elucidate the spatial structures of city networks. The three main conclusions drawn are generalized as follows:

(1) The macro-spatial patterns of Jiangsu's city networks, represented by expressway traffic flow, present clear regional differences. The cities with relatively good space connection and network density were distributed along the banks of the Yangtze River, and extended northward to the north boundary of Yangzhou, Taizhou, and Nantong. Furthermore, multiple different sub-network systems were identified as having internal development. Such distribution characteristics show that Jiangsu Province is not a complete economic regional unit. The fracturing of Jiangsu Province is likely due to it belonging to both the Yangtze River Delta Economic Zone, with Shanghai as the core, and the Huaihai Economic Zone, with Xuzhou as the core.

(2) City and city-dyad connection axles have clear hierarchical characteristics. Cities high in the regional hierarchy are relatively economically well-developed and wealthy, and are distributed along the Yangtze River, the provincial boundary, or the trunk expressways. This reflects that the importance of the city hierarchy and it's spatial coupling with the city's social economic attributes and geographical locations. This result is similar with Jiao's study by using the HSR (High-speed rail) lines of Chinese cities in two aspects [13]. One is in statistical, as the relationship between the degree of importance and rank order of cities in terms of centrality conformed to an exponential function [13]. The other is in geographical; the cities with high hierarchical rank were mainly located along trunk expressway lines in this paper, and they were mainly located along trunk HSR lines in Jiao's study [13]. This signifies that trunk transport lines can gradually outpace or change the hierarchy of cities. According to the city-dyads connection axle, their hierarchy and geographical distance have clear spatial interactions and superposition; the close connection within a short distance is a clear "localization", or could describe the functional connection inside a relatively complete regional system.

(3) Using community detection to explore city or urban network structures is a new method in city-region analysis $[24,38,39]$. Six spatially connected and clearly defined "city communities" are identified and four spatial metaphors are found in Jiangsu by using the Walktrap algorithm of community detection in our study, which is similar to Chen's study by using the Infomap algorithm of community detection for extracting spatial structure and organization patterns of China's city networks based on the highway passenger flows [39]. From the results, our study suggests that the community detection method would be a powerful analytical tool in the quantitative analysis of multi-scale city networks' spatial structure.

\subsection{Outlook}

The results of this study have important implications for providing a scientific basis for policy-making, so as to better promote social and economic development and improve the efficiency of economic space. The plan for urban space layout, as proposed in the existing Jiangsu Urban System Planning (2015-2030) ("Planning"), is to construct the urbanized spatial patterns of "one belt, two axles, three circles, and one pole", a City Belt along the Yangtze River, Coastal Urban Axle and Urban Axle along East Longhai, Nanjing, Xuzhou, and the Suzhou-Wuxi-Changzhou Metropolis Circles, and the Huai'an Growth Pole. In comparison with this Planning, this study proposes the following.

(1) Currently, the regions with a high degree of network development are mainly concentrated in the Nanjing Metropolis Circle, Suzhou-Wuxi-Changzhou Metropolis Circle, and Nantong Metropolis Circle (including Yancheng), within the scope of the Yangtze River Delta Region. Furthermore, recently, the Nantong Metropolis Circle has undergone swift and violent development. As one of the three metropolis circles that Jiangsu Province is developing, Xuzhou Metropolis Circle is relatively limited in terms of an internal network, and its network intensity is even lower 
than that of Nantong Metropolis Circle. Therefore, in the future, Xuzhou still needs to build internal strength and functional connections, raise its capability for radiation and agglomeration to surrounding regions, and drive the development of the entire Northern Jiangsu region.

(2) The Urban Axle along East Longhai is an important route of access to the sea in Midwestern China, and acts as an axle belt for driving the development of Northern Jiangsu. However, from existing urban spatial connection patterns, Xuzhou and Lianyungang in the Urban Axle along East Longhai do not show strong functional connection, and their inter-city connection degree is limited. Therefore, in the future, forming the development axle with Xuzhou and Lianyungang as center cities more quickly is necessary. This can be accomplished by relying on traffic corridors such as the Longhai-Lanxin Railway Transportation and Lianyungang-Xuzhou Special Passenger Railway Line.

(3) Historically, Jiangsu Province has lagged in coastal development, and the current development status of coastal cities is not in agreement with the province's excellent geographical locations. In this context, Jiangsu Province has clearly proposed a development strategy for constructing a coastal urban axle, attempting to facilitate the rapid development of coastal cities and accelerate regional economic integration construction. However, in practice, the spatial connection between NYC and LYGC in coastal areas is weak, and there are opportunities to improve the coastal urban axle.

(4) In comparison with Southern Jiangsu, the hinterland in Northern Jiangsu is still at the preliminary stage of economic development. Furthermore, the difference in development across the region is large, as embodied in the Huai'an and Suqian regions. The HSC has been recognized in previous sections as a low-value region for regional economic development. Therefore, promoting the development of the Huai'an Growth Pole and strengthening the functional connection of HSC with other communities is an important consideration in the future development of Jiangsu Province. This would facilitate significant regional development and change the current state of diffused urban space organization and imbalanced regional development in Jiangsu Province.

Acknowledgments: This research was funded by the National Natural Science Foundation of China (grant number 41601165, 41430635, 41571379), National Natural Science Foundation of Fujian Province, China (grant number 2016J05093), and China Postdoctoral Science Foundation Funded Project (grant number 2016M590588).

Author Contributions: Wenqian Ke designed the analytical framework. Wenqian Ke and Zhaoyuan Yu analyzed the data. Wei Chen contributed analysis tools and methods. Wenqian Ke and Wei Chen wrote the paper. All authors read and approved the final manuscript.

Conflicts of Interest: The authors declare no conflict of interest.

\section{References}

1. Castells, M. Globalisation, networking, urbanisation: Reflections on the spatial dynamics of the information age. Urban Stud. 2010, 47, 2737-2745. [CrossRef]

2. Castells, M. The Rise of the Network Society; Blackwell: Oxford, UK, 1996; pp. 132-134.

3. Batty, M. Urban Information Networks: The Evolution and Planning of Computer Communications Infrastructure. In Cities of the 21st Century New Technologies and Spatial System; Brotchie, J., Batty, M., Hall, P., Eds.; Longman Cheshire: Boulder, CO, USA, 1991; pp. 65-72.

4. Taylor, P. Urban hinterworlds: Geographies of corporate service provision under conditions of contemporary globalization. Geography 2001, 86, 51-60.

5. Taylor, P.; Hoyler, M.; Verbruggen, R. External urban relational process: Introducing central flow theory to complement central place theory. Urban Stud. 2010, 47, 2803-2818. [CrossRef]

6. Taylor, P.; Catalano, G.; Walker, D.R.F. Measurement of the world city network. Urban Stud. 2002, 39, 2367-2376. [CrossRef]

7. Taylor, P. Specification of the world city network. Geogr. Anal. 2001, 33, 181-194. [CrossRef]

8. Camagni, R.; Salone, C. Network Urban Structures in Northern Italy: Elements for a Theoretical Framework. Urban Stud. 1993, 30, 1053-1064. [CrossRef] 
9. Scott, A.J.; Agnew, J.; Soja, E.W. Global City-Regions: Trends, Theory, Policy; Oxford University Press: Oxford, UK, 2001; pp. 326-327.

10. Hall, P. Planning for the mega-city: A new eastern Asian urban form? In East West Perspectives on 21st Century Urban Development: Sustainable Eastern and Western Cities in the New Millennium; Brotchie, J., Newton, P., Hall, P., Eds.; Ashgate: Aldershot, UK, 1999; pp. 3-36.

11. Hall, P.; Pain, K. The Polycentric Metropolis: Learning from Mega-City Regions in Europe; Taylor \& Francis Group: Routledge, UK, 2006; pp. 384-385.

12. Derudder, B.; Witlox, F. Mapping world city networks through airline flows: Context, relevance, and problems. J. Transp. Geogr. 2008, 16, 305-312. [CrossRef]

13. Jiao, J.; Wang, J.; Jin, F. Impacts of high-speed rail lines on the city network in China. J. Transp. Geogr. 2017, 60, 257-266. [CrossRef]

14. Limtanakool, N.; Schwanen, T.; Dijst, M. Developments in the Dutch urban system on the basis of flows. Reg. Stud. 2009, 43, 179-196. [CrossRef]

15. Li, J.; Ye, Q.; Deng, X.; Liu, Y.; Liu, Y. Spatial-temporal analysis on Spring Festival travel rush in China based on multisource big data. Sustainability 2016, 8, 1184. [CrossRef]

16. Malecki, E.J. The economic geography of the Internet's infrastructure. Econ. Geogr. 2002, 78, 399-424. [CrossRef]

17. Mitchelson, R.L.; Wheeler, J.O. The flow of information in a global economy: The role of the American urban system in 1990. Ann. Assoc. Am. Geogr. 1994, 84, 87-107. [CrossRef]

18. Chen, W.; Xiu, C.L.; Liu, W.D.; Yu, Z.Y. Featured graphic. Visualizing intercity scientific collaboration networks in China. Environ. Plan. A 2015, 47, 1-3. [CrossRef]

19. Taylor, P. The new geography of global civil society: NGOs in the world city network. Globalizations 2004, 1, 265-277. [CrossRef]

20. Gutierrez, J.; Gonzalez, R.; Gomez, G. The European high speed train network: Predicted effects on accessibility patterns. J. Transp. Geogr. 1996, 4, 227-238. [CrossRef]

21. Li, S.M.; Shum, Y.M. Impacts of the national trunk highway system on accessibility in China. J. Transp. Geogr. 2001, 9, 39-48. [CrossRef]

22. Amaral, L.A.N.; Scala, A.; Barthélémy, M. Classes of small-world networks. Proc. Natl. Acad. Sci. USA 2000, 97, 11149-11152. [CrossRef] [PubMed]

23. Bagler, G. Analysis of the airport network of India as a complex weighted network. Physica A 2008, 387, 2972-2980. [CrossRef]

24. Zhou, M.; Yue, Y.; Li, Q.Q. Portraying temporal dynamics of urban spatial divisions with mobile phone positioning data: A complex network approach. Int. J. Geo-Inf. 2016, 5, 1-21. [CrossRef]

25. Dash, N.G.; Rae, A. An economic geography of the United States: From commutes to megaregions. PLoS ONE 2016, 11, e0166083.

26. Beaverstock, J.V.; Smith, R.G.; Taylor, P. World-city network: A new metageography? Ann. Assoc. Am. Geogr. 2000, 90, 123-134. [CrossRef]

27. Girvan, M.; Newman, M.E.J. Community structure in social and biological networks. Proc. Natl. Acad. Sci. USA 2002, 99, 7821-7826. [CrossRef] [PubMed]

28. Clauset, A.; Newman, M.E.J.; Moore, C. Finding community structure in very large networks. Phys. Rev. E 2004, 70, 066111. [CrossRef] [PubMed]

29. Pons, P.; Latapy, M. Computing communities in large networks using random walks. J. Graph Algorithms Appl. 2006, 10, 191-218. [CrossRef]

30. Blondel, V.D.; Guillaume, J.L.; Lambiotte, R. Fast unfolding of communities in large networks. J. Stat. Mech. 2008, 2008, P10008. [CrossRef]

31. Raghavan, U.N.; Albert, R.; Kumara, S. Near linear time algorithm to detect community structures in large-scale networks. Phys. Rev. E 2007, 76, 036106. [CrossRef] [PubMed]

32. Rosvall, M.; Bergstrom, C.T. Maps of random walks on complex networks reveal community structure. Proc. Natl. Acad. Sci. USA 2008, 105, 1118-1123. [CrossRef] [PubMed]

33. Steinhaeuser, K.; Chawla, N.V. Identifying and evaluating community structure in complex networks. Pattern Recognit. Lett. 2010, 31, 413-421. [CrossRef]

34. Yang, Z.; Algesheimer, R.; Tessone, C.J. A comparative analysis of community detection algorithms on artificial networks. Sci. Rep. 2016, 6, 30750. [CrossRef] [PubMed] 
35. Zipf, G.K. Human Behavior and the Principle of Least Effort; Addison-Wesley: Cambridge, UK, 1949; p. 69.

36. Kanungo, T.; Mount, D.; Netanyahu, N.; Piatko, C. An efficient K-means clustering algorithm: Analysis and implementation. IEEE Trans. Pattern Anal. Mach. Intell. 2000, 24, 881-892. [CrossRef]

37. Christaller, W. Die Zentralen Orte in Süddeutschland; Gustav Fischer Verlag: Jena, German, 1933; pp. 68-124.

38. Jiao, J.J.; Wang, J.E.; Jin, F.J.; Wang, H. Impact of high-speed rail on inter-city network based on the passenger train network in China, 2003-2013. Acta Geogr. Sin. 2016, 71, 265-280. (In Chinese)

39. Chen, W.; Liu, W.D.; Ke, W.Q.; Wang, N.Y. The spatial structure and organization patterns of China's city networks based on the highway passenger flows. Acta Geogr. Sin. 2017, 72, 224-241. (In Chinese)

(C) 2017 by the authors. Licensee MDPI, Basel, Switzerland. This article is an open access article distributed under the terms and conditions of the Creative Commons Attribution (CC BY) license (http://creativecommons.org/licenses/by/4.0/). 